\title{
A Versatile AIE Fluorogen with Selective Reactivity to Primary Amines for Monitoring Amination, Protein Labeling and Mitochondrial Staining
}

\author{
Xinyuan He, ${ }^{[a,+]}$ Huilin Xie, ${ }^{[a,+]}$ Lianrui Hu, ${ }^{[a,+]}$ Pengchao Liu, ${ }^{[b]}$ Changhuo Xu, ${ }^{[a]}$ Wei He, ${ }^{[a]}$ Wutong Du, ${ }^{[a]}$ \\ Siwei Zhang, ${ }^{[a]}$ Hao Xing, ${ }^{\left[{ }^{[a]}\right.}$ Xinyue Liu, ${ }^{[a]}$ Hojeong Park, ${ }^{[a]}$ Tsz Shing Cheung, ${ }^{[a]}$ Min-Hui Li, ${ }^{[c]}$ Ryan T. K. \\ Kwok, ${ }^{[a, d]}$ Jacky W. Y. Lam, ${ }^{[a, d]}$ Jian Lu ${ }^{*},[b]$ and Ben Zhong Tang ${ }^{*,[a, d, e]}$
}

[a] X. He, H. Xie, L. Hu, C. Xu, W. He, W. Du, S. Zhang, H. Xing, X. Liu, H. Park, T. S. Cheung, Dr. R. T. K. Kwok, Dr. J. W. Y. Lam, Professor B. Z. Tang Department of Chemistry, Hong Kong Branch of Chinese National Engineering Research Center for Tissue Restoration and Reconstruction, State Key Laboratory of Molecular Neuroscience, Division of Life Science and Department of Chemical and Biological Engineering, The Hong Kong University of Science and Technology, Clear Water Bay, Kowloon, Hong Kong, China

E-mail: tangbenz@cuhk.edu.cn

[b] P. Liu, Professor J. Lu

Department of Mechanical Engineering, City University of Hong Kong, Greater Bay Joint Division, Shenyang National Laboratory for Materials Science, Tat Chee Avenue, Kowloon, Hong Kong, China

E-mail: jianlu@cityu.edu.hk

[c] M. H. Li

Chimie ParisTech PSL University Paris, CNRS, Institut de Recherche de Chimie paris 75005, Paris, France

[d] Dr. R. T. K. Kwok, Dr. J. W. Y. Lam, Professor B. Z. Tang

Guangdong-Hongkong-Macou Joint Laboratory of Optoelectronic and Megnetic Functional Materials

[e] Professor B. Z. Tang

Center for Aggregation-Induced Emission, SCUT-HKUST Joint Research Institute, State Key Laboratory of Luminescent Materials and Devices, South

China University of Technology, Guangzhou 510640, China; Shenzhen Institute of Aggregate Science and Technology, School of Science and Engineering, The Chinese University of Hong Kong, Shenzhen, 2001 Longxiang Boulevard, Longgang District, Shenzhen City, Guangdong 518172, China

[+] These authors contributed equally to this work

Supporting information for this article is given via a link at the end of the document.

\begin{abstract}
Specific bioconjugation for native primary amines is highly valuable for both chemistry and biomedical research. Despite all the efforts, scientists lack a proper strategy to achieve high selectivity for primary amines, not to mention the requirement of fast response for real applications. Herein, in this work, we report a chromone-based aggregation-induced emission (AIE) fluorogen called CMVMN as a self-reporting bioconjugation reagent for selective primary amine identification, and its applications for monitoring bioprocesses of amination and protein labeling. CMVMN is AIE-active and is capable of solid-state sensing. Thus, its electrospun films are manufactured for visualization of amine diffusion and leakage process. CMVMN also shows good biocompatibility and potential mitochondria-staining ability, which provides new insight for organelle-staining probe design. Combined with its facile synthesis and good reversibility, CMVMN not only shows wide potential applications in biology, but also offers new possibilities for molecular engineering.
\end{abstract}

\section{Introduction}

In-situ probe of biomolecules has become a new pursuit for scientists due to its increasing impact on studying cellular processes and therapeutic applications. ${ }^{1}$ The assignment of the 2008 Nobel Prize in Chemistry to genetically encoded fluorescent proteins, which is specific for dynamic tracking of protein, is a good example. ${ }^{2}$ However, cellular systems are full of biological electrophiles and nucleophiles, and no such tools as fluorescent proteins can be used for monitoring nonproteinaceous targets in their native habitat. ${ }^{3}$ To deal with this problem, orthogonal bioconjugation, which was coined by Bertozzi and coworkers in $2013^{4}$ and refers to selective chemical reactions that can enact rapidly in a living system and meanwhile be inert to native biomolecules has been developed. ${ }^{5}$ Despite of the fabulous success in selective labelling of biomolecules in-situ based on bioorthogonal ractions, ${ }^{6}$ the prefunctionalization of nature substrate with one of the specific reaction handles like alkynes, azides, ketones and tetrazines is complex, which limits its application scope. ${ }^{7}$ To monitor the time course of the reaction in a real-time manner, an additional indicator is further needed. ${ }^{8}$ On the other hand, by means of the advancement of synthetic organic chemistry, it is possible and meaningful to utilize native functional groups as "handles" and self-reporters for in-situ and selective bioconjugation.

Amines are ubiquitous in living systems, such as neurotransmitters, nucleic acids, amino acids, etc., ${ }^{9}$ making them ideal native candidates for bioconjugation. Nonetheless, the simple and worldwide covalent labelling of amines with compounds bearing isothiocyanate, ${ }^{10} \mathrm{~N}$-hydroxysuccinimidyl ester, ${ }^{11}$ or pen-tafluorophenyl ${ }^{12}$ is not applicable for selective bioconjugation because of the similar reactivity to primary amines and secondary amines. Previously, primary amines were reported to show special reactivity towards some electrophiles (such as aldehyde, pyrylium and ylidenemalononitrile enamines) and light-induced oxidative coupling reagent (such as onitrobenzyl alcohols and azidophenols), ${ }^{13}$ which made it even more attractive as a native "handle". Notably, fast and efficient bioconjugation of activated alkynes and native amines without prefunctionalization and by-product generation (to minimize the disturbance to biosystem) was reported by our group. ${ }^{14}$ Encouraged by this success, we are eager to create probes with high affinity, reactivity and selectivity towards primary amines.

Chromone is reported to be reactive to amines at high concentration or high temperature. Interestingly, its products with primary amines form a special intramolecular hydrogen 
bond that contributes to the high molecular stability. This possibly induces a high affinity of chromone towards primary amines. ${ }^{15}$ The introduction of a methyl group in a position of the carbonyl group of chromone can further affect its reaction with secondary amines to result in enhanced selectivity towards primary amines. ${ }^{16}$ Besides, the discovery of epicocconone, which shows a relatively high reactivity to primary amines and is capable of staining proteins in the aqueous media, reminds us the importance of rational molecular design. ${ }^{17}$ So even though none of the reported chromone derivatives can realize fast, selective bioconjugation for primary amines at room temperature, ${ }^{15,16,18}$ we believe that their selectivity can be enhanced through molecular engineering. Specifically, when an electron withdrawing group is introduced to the a position of chromone, the obtained new compound is activated and may show a good affinity and selectivity towards primary amines. Or more, with a similar donor-acceptor structure as epicocconone, the fluorescent signal may be seen as well.

Fluorescence sensing is a non-invasive imaging modality with easy operation and high spatial-temporal resolution and is a hotspot in recent years. ${ }^{19}$ Traditional organic fluorescent probes tend to aggregate in aqueous solution due to their high hydrophobicity, which triggers the undesirable aggregation caused quenching effect. ${ }^{20}$ Fortunately, the discovery of a phenomenon of aggregation-induced emission (AIE) has solved the problem. ${ }^{21}$ Molecules with AIE effect are non-emissive or weakly emissive in the molecularly dissolved state, but become highly emissive in the aggregate state. This unique photophysical property makes them as ideal candidates for the observation of bioconjugation procedure. ${ }^{22}$ Conceivably, if an AIE fluorogen that undergoes specific reaction readily with primary amines is developed, its applications will no longer be limited to diluted solutions.

In this work, we introduced a one-step synthesis of (E)-2-\{5,5dimethyl-3-[2-(4-oxo-4H-chromen-3-yl)vinyl]cyclohex-2-en-1-

ylidene\}malononitrile (abbreviated as CMVMN, Scheme 1) which could undergo selective bioconjugation with primary amines, and showed self-reporting property. The reaction of CMVMN with primary amines was fast and highly selective. Meanwhile, a distinct color change and fluorescence turn-on signal at $595 \mathrm{~nm}$ were both detected, enabling CMVMN to monitor the bioprocesses of amination and protein labeling. NMR titration and theoretical calculation were also conducted and verified the reaction mechanism. CMVMN was AIE-active and was capable of solid-state sensing. Thus, its electrospun films were fabricated for visualization of amine diffusion and leakage process in a real-time manner. Furthermore, CMVMN showed good biocompatibility and a mitochondria-staining ability for cell imaging.

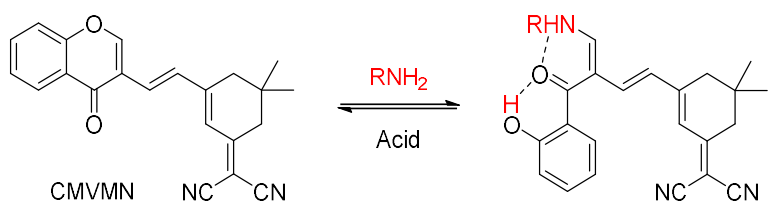

Scheme 1. Specific and reversible reaction of CMVMN with primary amines.

\section{Results and Discussion}

The photophysical properties of CMVMN were first investigated. CMVMN showed absorption maximum at $387 \mathrm{~nm}$ (Figure S5) and weak emission in THF solution at $530 \mathrm{~nm}$ with a quantum yield (QY) of $0.3 \%$ (Figure 1a). When a poor solvent of deionized water was added to the THF solution, an enhancement in fluorescence intensity was observed due to the aggregation of CMVMN molecules. At water fraction of $95 \%$, the emission peak was located at $550 \mathrm{~nm}$ with a QY of $0.7 \%$, which verified the AIE property of CMVMN. However, the fluorescence signal was still very weak and was similar to that of amorphous powder with a QY of $0.5 \%$ (Figure $1 \mathrm{~b}$ and Figure S6a). To get indepth knowledge, we placed the amorphous powder of CMVMN into an NMR tube and then cooled the set-up down. As expected, distinct orange fluorescence was observed at $195 \mathrm{~K}$, whose intensity became stronger at $77 \mathrm{~K}$ (Figure 1b). This indicated the molecule became more emissive once its intramolecular motion was slowed down at low temperature.

Interestingly, the single crystal of CMVMN was highly emissive (Figure 1c and Figure S6b) and its QY (63\%) was 126 times higher than that of amorphous powder. Such a phenomenon was observed before and was named as "crystallization-induced emission". ${ }^{23}$ For CMVMN crystals, each molecule was found to form six hydrogen bonds with surround molecules, which efficiently restricted its intramolecular motion (Figure 1d). The strong intermolecular interactions in the crystal largely rigidified the molecular conformation and avoided detrimental $\pi-\pi$ interactions, making them show intense crystal-state emission. ${ }^{24}$

The emission maximum in the crystalline state $\left(\lambda_{\mathrm{em}}=530 \mathrm{~nm}\right.$, $\tau=2.12 \mathrm{~ns}, 77 \mathrm{~K})$ was blue-shifted compared with that of the amorphous state $\left(\lambda_{\mathrm{em}}=605 \mathrm{~nm}, \tau=1.06 \mathrm{~ns}, 77 \mathrm{~K}\right)$ (Figure S7 and S8). Probably, the loose packing in the amorphous state enabled the molecules to undergo some sort of intramolecular motion and hence an excited-state minimum with a small band gap emerged for amorphous sample. Eventually, the red-shifted and weakened emission was observed (see the proposed decay pathways in Figure 1e). ${ }^{25}$ The red-shifted and decreased emission of the crystalline solid in $298 \mathrm{~K}$ was also in accordance with this conjecture (Figure 1c and Figure S7a).
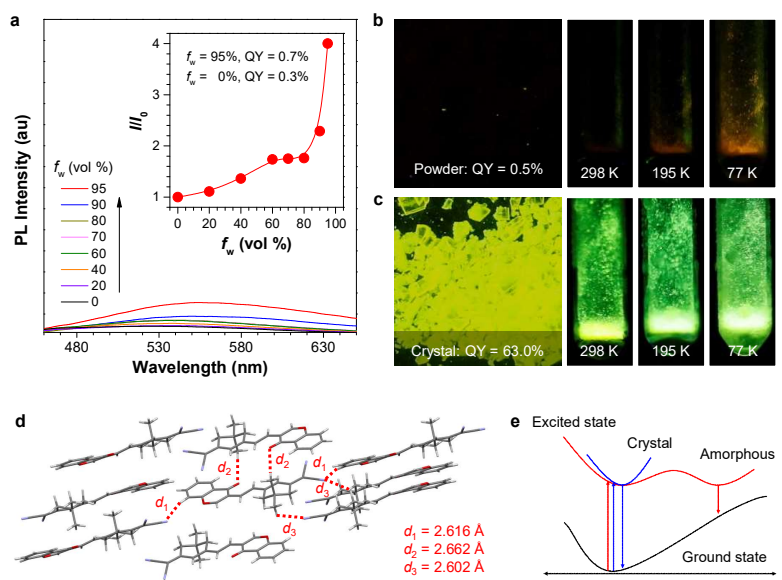

Figure 1. (a) Photoluminescence (PL) spectra of CMVMN $(10 \mu \mathrm{M})$ in THF/water mixtures with different water fractions $\left(f_{\mathrm{w}}\right)$. Inset: Plot of $I / I_{0}$ vs. $f_{\mathrm{w}} ; I$ $=\mathrm{PL}$ intensity at $f_{\mathrm{w}}, l_{0}=\mathrm{PL}$ intensity at $f_{\mathrm{w}}=0 \%$. $\lambda_{\text {ex }} / \mathrm{em}=387 / 550 \mathrm{~nm}$. Fluorescent images of (b) amorphous powder and (c) crystalline solid of CMVMN taken under UV irradiation at $298 \mathrm{~K}, 195 \mathrm{~K}$ and $77 \mathrm{~K}$. (d) Crystal packing of CMVMN molecules. (e) Proposed decay pathways along potential energy surfaces of CMVMN in the crystalline and amorphous states. 

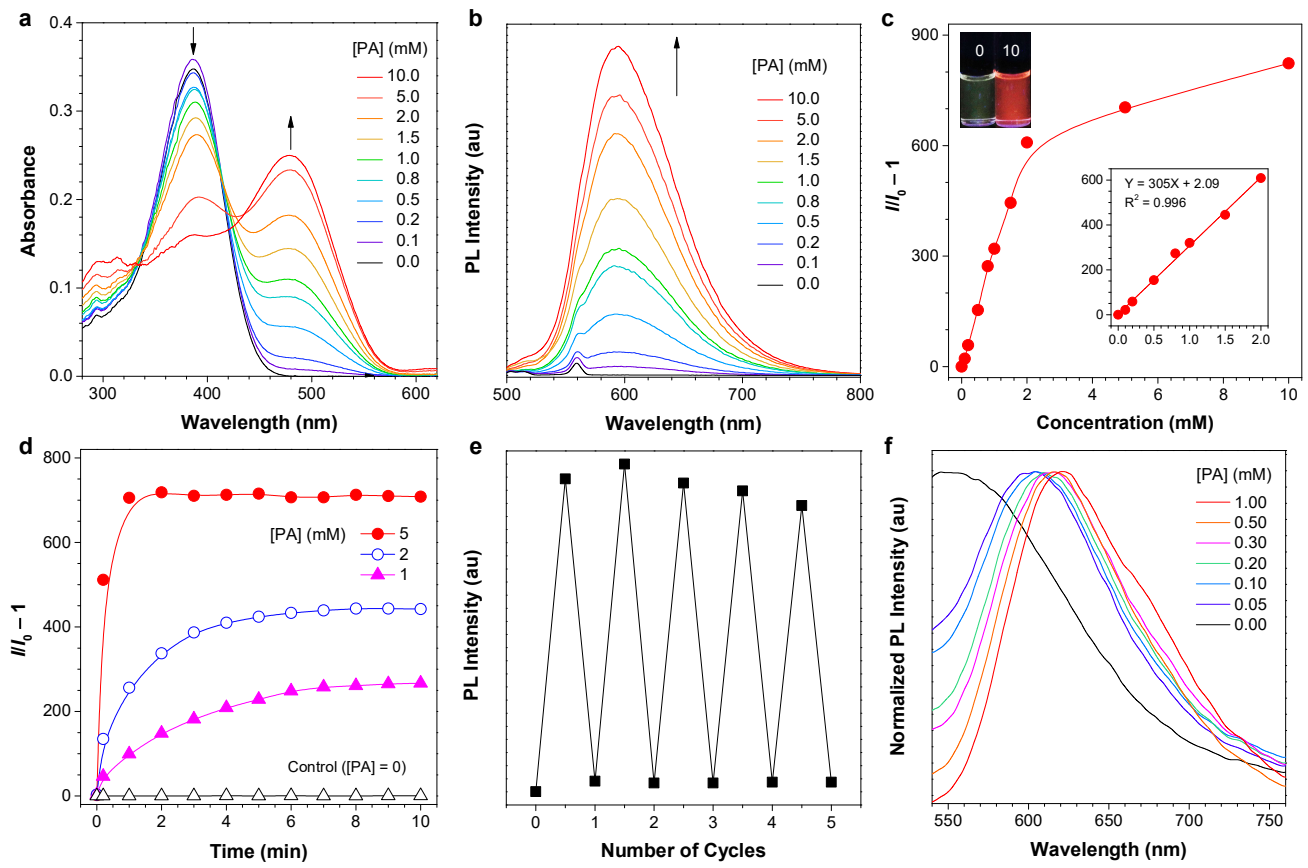

Figure 2. Changes in (a) absorption and (b) PL spectra of CMVMN (10 $\mu \mathrm{M})$ with addition of PA in THF solution. (c) Plot of relative fluorescence intensity (I//0 - 1) vs. concentration of PA [PA]. Inset: (upper) Photographs of CMVMN before and after reaction with $10 \mathrm{mM}$ PA taken under UV irradiation; (lower) linear fitting curve of $\left(I / I_{0}-1\right)$ toward $[P A]$. (d) Time course of amination reaction of CMVMN by PA. (e) Reversible changes in PL intensity of CMVMN upon repeated exposures to PA and trifluoroacetic acid. (f) Normalized PL spectra of spin-coating films of CMVMN fumed with different amount of PA for 1 min. $\lambda_{\text {ex/em }}=480 / 595$ $\mathrm{nm}$.

The reaction of CMVMN with primary amines was studied via the spectroscopy method and propylamine (PA) was chosen as a model compound. Upon addition of PA to a THF solution of CMVMN, the absorption peak at $387 \mathrm{~nm}$ decreased, but a new peak emerged at $480 \mathrm{~nm}$ (Figure 2a). Because the new absorption peak was located in the visible light region, direct visualization of the amination process became possible. Meanwhile, a significant fluorescence turn-on signal was detected at $595 \mathrm{~nm}$ and the fluorescence intensity increased by 823 folds at a [PA] of $10 \mathrm{mM}$, which enabled the sensitive detection of PA (Figure 2b). After that, quantitative monitoring of $P A$ was performed and a good linear curve between the relative fluorescence intensity $\left(I / I_{0}-1\right)$ and the PA concentration $(0-2.0$ $\mathrm{mM}$ ) was obtained (Figure $2 \mathrm{c}$ ). The detection limit $(k=3)$ was found to be $4.4 \mu \mathrm{M},{ }^{26}$ indicating the good sensitivity. The PA detection could be realized via a colorimetric method (Figure S9) as well. Hence, CMVMN could be used not only for the detection of primary amines, but also as a fluorescent and colorimetric dual-mode probe.

Because the dynamics of the amination process is a worth knowing story, we thus followed the time course of the reaction. As shown in Figure $2 \mathrm{~d}$, the reaction initiated rapidly and the fluorescence signal almost reached a plateau within 5 min even at a low PA concentration $(1.0 \mathrm{mM})$. The signal saturation time was further reduced to $1 \mathrm{~min}$ at a high PA concentration (5.0 $\mathrm{mM}$ ). Obviously, the fast amination would be very valuable for real applications. On the other hand, nucleophilic reactions were found to be better carried out under alkaline conditions. ${ }^{27}$ Thus, the autocatalytic effect of PA at high concentration could be illustrated. However, if PA was protonated in the presence of trifluoroacetic acid (TFA), the amination process should be inhibited to result in decreased fluorescence. In this work, although the amination was well finished before the addition of TFA, decreased fluorescence intensity was also observed, revealing the good reversibility of the amination process (Figure $2 \mathrm{e})$. Even more, the fluorescence turned out to be controllable via cycle addition of PA or TFA (Figure $2 \mathrm{e}$ ), making CMVMN a promising tool for long-time dynamic tracking. ${ }^{28}$

Solid-state sensing is much preferred than liquid phase sensing in real applications thanks to its simple sample preparation. ${ }^{29}$ Because AIEgens are highly luminescent in the solid-state, they are ideal candidates for such purpose. ${ }^{30}$ Curiously, we further fabricated the spin-coating films of CMVMN from its dichloromethane solution and fumed it with PA vapors. As depicted in Figure $2 f$, after exposure to PA vapors for $1 \mathrm{~min}$, a remarkable red-shift in the emission peak was observed. It was worth noting that the emission peak red-shifted by $50 \mathrm{~nm}$ after exposure to PA vapors of low concentration $(0.05 \mathrm{mM})$, indicating the high sensitivity of CMVMN to PA vapors in the solid-state. Besides, quantitative detection of PA vapors using the spin-coating films was performed (Figure S10). Moreover, CMVMN exhibited good stability and reversibility after repeated exposure to PA and trifluoroacetic acid vapors, as evidenced by the similar spectrum to that of the initial sample form the ${ }^{1} \mathrm{H}$ NMR measurement (Figures S1 and S11).

In addition, the fluorescence response of CMVMN to various nucleophiles bearing amido, hydroxyl or sulfhydryl groups was investigated. The results showed that CMVMN reacted not only with PA, but also other primary amines including ethanolamine, butylamine, amylamine, cyclohexylamine, benzylamine and tertbutylamine (TBA), as revealed by the emission enhancement effect of these molecules on CMVMN (Figure 3). The steric effects of amines played a decisive role on their reactivity because of the obvious decrease in their fluorescence enhancement effect by increasing the steric hindrance (TBA > benzylamine $>$ cyclohexylamine). If CMVMN was incubated with 


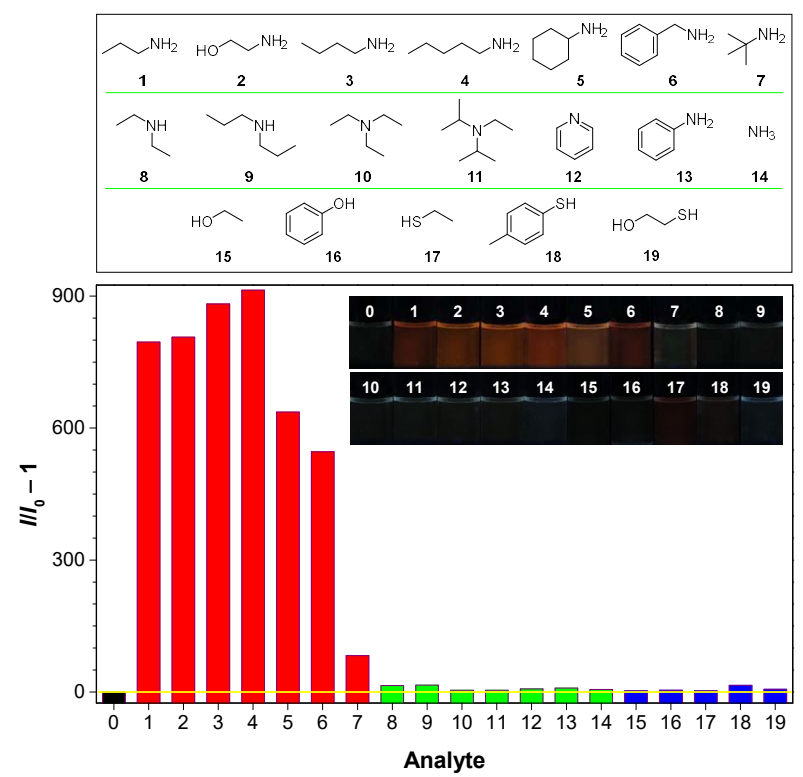

Figure 3. (Upper panel) Chemical structures of the analytes investigated in this study. (Lower panel) Relative fluorescence intensity $\left(I / I_{0}-1\right)$ of CMVMN $(10 \mu \mathrm{M})$ after its mixing with different analytes $(10 \mathrm{mM})$ in THF for $10 \mathrm{~min}$. Analytes: [(0) CMVNM (control)], (1) PA, (2) ethanolamine, (3) butylamine, (4) amylamine, (5) cyclohexylamine, (6) benzylamine, (7) TBA, (8) diethylamine, (9) dipropylamine, (10) trimethylamine, (11) N, N-diisopropylethylamine, (12) pyridine, (13) aniline, (14) ammonia, (15) ethanol, (16) phenol, (17) ethanethiol (18) $p$-toluenethiol, (19) mercaptoethanol. $\lambda_{\text {ex/em }}=480 / 595 \mathrm{~nm}$. Inset: fluorescent images of the mixtures of the analytes and CMVMN.

TBA for a long time, only a gradual but slow increase in fluorescence was detected (Figure S12), which again proved the steric effect-caused weak reactivity. Under the same conditions, the fluorescence enhancement effect of primary amines was much bigger than that of other amines such as secondary amines, tertiary amines, heterocyclic amines, aromatic amines and ammonia. Meanwhile, nucleophiles with hydroxyl or sulfhydryl showed no strong effect on the fluorescence of CMVMN. These results revealed the high reactivity and selectivity of CMVMN towards primary amines.

To get a deeper insight into the amination process, NMR titration experiments were further conducted. According to the previous report, the ${ }^{1} \mathrm{H}$ NMR spectra of enamines generated from chromones and primary amines showed a characteristic broad signal at $\delta 9-12$, which attributed to the resonance of an $\mathrm{NH}$ group forming a strong intermolecular hydrogen bond. ${ }^{15}$ Accordingly, a new broad peak at $\delta 10.8$ emerged in the ${ }^{1} \mathrm{H}$ NMR spectra of CMVMN after addition of PA and the signal was intensified when more PA was added (Figure S13). Meanwhile, the original conjugated structure of CMVMN was destroyed to result in a decreased deshielding effect on the surrounding hydrogen atoms. As expected, new peaks appeared at lower fields compared with those of CMVMN. Beyond that, the HRMS spectra of the reaction solution was measured and a mass peak at mass-to-charge ratio $(\mathrm{m} / \mathrm{z})$ of $424.2002[\mathrm{M}+\mathrm{Na}]^{+}$(Figure S14) related to compound S7 (Scheme 2) was detected. Hereby, CMVMN may follow a reaction path similar to typical chromones. ${ }^{15,16}$

Later, density functional theory calculation was carried out and the results were in line with the experimental results. The energy profile for the reaction pathway of CMVMN with PA was shown in Scheme 2. The initial nucleophilic attack followed by isomerization formed the intermediate product S3 with a high energy $(10.1 \mathrm{kcal} / \mathrm{mol})$. Later, the rate-limiting step of bond dissociation taken place accompanied with isomerization to form a metastable intermediate product $\mathrm{S} 5(3.0 \mathrm{kcal} / \mathrm{mol})$. Since the reaction energy barrier was only $20.7 \mathrm{kcal} / \mathrm{mol}$, the amination of CMVMN with primary amines could happen readily at room temperature. Importantly, the obtained products were stabilized by intramolecular hydrogen bonds. Thus, it was stable with low energy $(-4.8 \mathrm{kcal} / \mathrm{mol})$.

However, the story for secondary amines was different. Diethylamine was chosen as a model compound here (Scheme S2) and the reaction energy barrier for bond dissociation was found to be much higher $(29.2 \mathrm{kcal} / \mathrm{mol})$ because of steric effect. As a result, its reaction with CMVMN usually ended after the generation of $S 9(12.3 \mathrm{kcal} / \mathrm{mol})$. But even if $\mathrm{S} 12$ could be produced, it was unstable, and its energy would be relatively higher $(5.1 \mathrm{Kcal} / \mathrm{mol})$ than that of $\mathrm{S} 7$ due to the lack of the stabilization effect by the intramolecular hydrogen bond formed by the carbonyl and $\mathrm{NH}$ groups. By the way, both S3 and S9 were not stable in view of their high energy. Apparently CMVMN

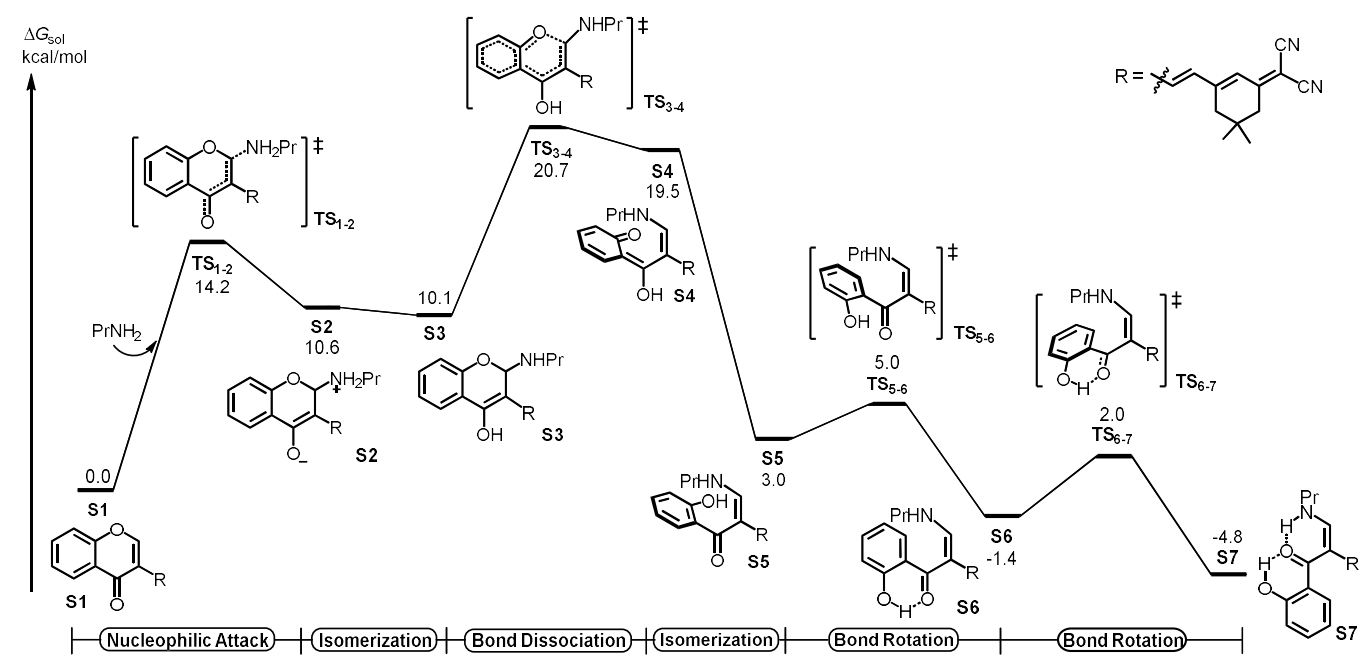

Scheme 2. Energy profile for the reaction pathway of CMVMN with PA. 
a

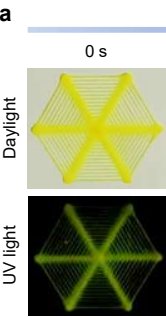

b

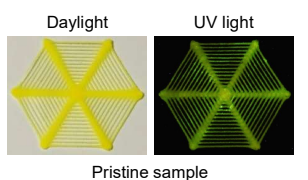

Pristine sample

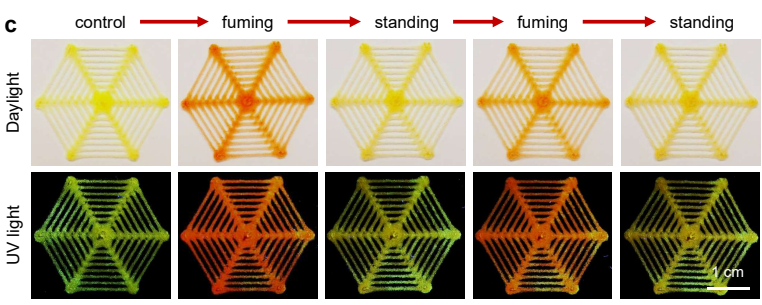

Figure 4. Photographs and fluorescence images of (a) the electrospun films of "spider-web" sensors printed from CMVMN microcrystals blended into polycaprolactone matrix upon exposure to PA vapor, (b) demonstration of the CMVMN sensors for the detection of PA leakage (arrow indicating the leaking point). (c) Reversible changes in absorption and emission colors during repeated cycling exposure to PA vapor.

is not a good reagent for one-step nucleophilic addition as other reported electrophiles. ${ }^{10-12}$ Overall, the steric effect of secondary amines impeded their reaction with CMVMN, while the special intramolecular hydrogen bonding in the resulting product contributed to the selective bioconjugation and reaction of CMVMN with primary amines.

Inspired by the good performance of spin-coating films of CMVMN for solid-state sensing, we further applied the present molecule to printing and used its electrospun films to manufacture "spider-web" sensors (Figure S15a). Thermogravimetric analysis of CMVMN was carried out which displayed a relatively high thermal decomposition temperature of $T_{d}=$ $294{ }^{\circ} \mathrm{C}$ (Figure S16), demonstrating the high thermostability of the molecule. The scanning electron microscopic images showed that the sensor was made up of micro fibers (Figure $\mathrm{S} 15 \mathrm{~b}$ ) and its loose structure was in favor of the fluxion of amine vapors. At the beginning, the sensor was pale yellow, showing yellow-green fluorescence. When fumed with PA, it became orange and emitted a red fluorescence (Figure S17). Obviously, this noticeable change would offer a workable method for equipment-free monitoring of primary amines. In addition, the color and emission of sensors remained unchanged upon exposure to diethylamine and trimethylamine vapors at the same conditions.

Later, real-time monitoring of PA was conducted. As shown in Figure 4a, CMVMN showed a fast and sensitive response to PA vapors. At the moment PA was added to the container, the color change was detected under both daylight and UV light. Notably, the color change first took place near the container and 30s later the whole sensor changed to orange (see more details in video S2-S3). Clearly, due to the high sensitivity of CMVMN and fast rate of the amination, the diffusion process of PA was successfully visualized and recorded. Further, a PA leakage model was constructed, in which PA was sealed in a culture dish
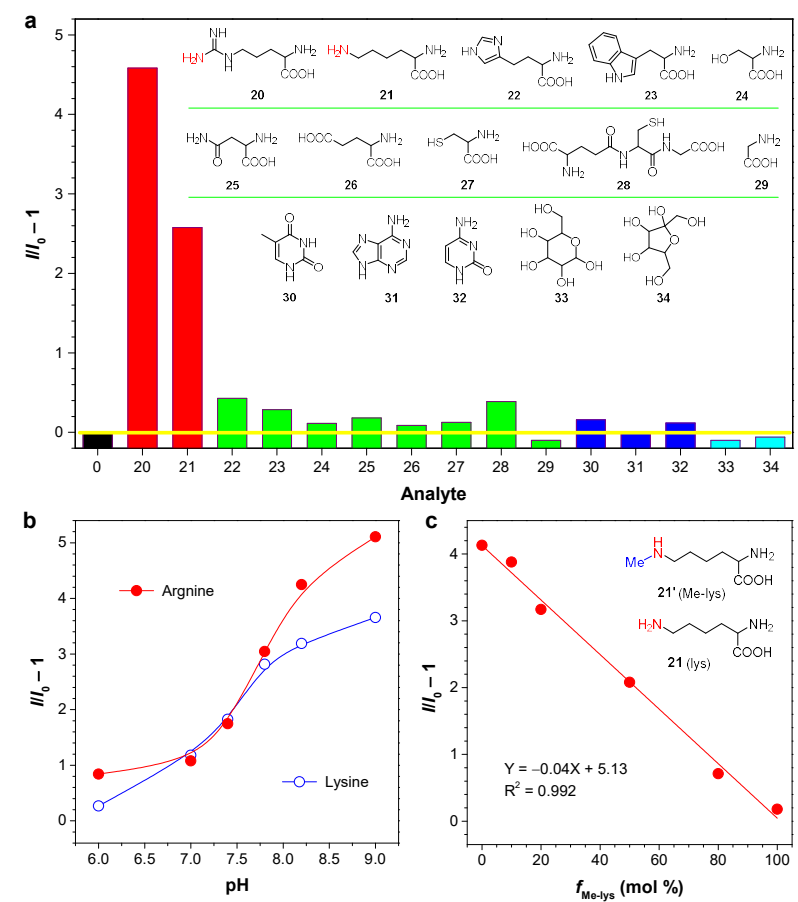

Figure 5. (a) Relative fluorescence intensity $\left(/ / I_{0}-1\right)$ of CMVMN $(10 \mu \mathrm{M})$ after mixing with various analytes $(10 \mathrm{mM})$ in PBS for $10 \mathrm{~min}$. Analytes: [(0) CMVMN (control)], (20) arginine, (21) lysine, (22) histidine, (23) tryptophan, (24) serine, (25) asparagine, (26) glutamic acid, (27) cysteine, (28) glutathione, (29) glycine, (30) thymine, (31) adenine, (32) cytosine, (33) glucose, (34) fructose. $\lambda_{\text {ex/em }}=480 / 595 \mathrm{~nm}$. (b) Effect of $\mathrm{pH}$ on the reaction of CMVMN with arginine (red solid circle) and lysine (blue open circle). (c) Change in fluorescence intensity of CMVMN against the fraction of $N_{6}$-Methyl-L-lysine (Me-lys) in a mixture of lysine and Me-lys.

and the sensor was put on top of it. Then, a leaking point was made by pierce with a needle and the whole process was recoded. As shown in Figure 4b and video S4-S5, a real-time leaking process was visualized, and the leaking point could be recognized easily. These results demonstrated the value of CMVMN for monitoring of leakage.

The reversibility of the manufactured devices was a concern since single-used materials often brought high costs and pollution. To check whether CMVMN could restore to its original state after detection, the fumed sensor was collected for further study. Excitingly, if we took the fumed sensor out and put it in open air, a gradual color fading was observed. It took about 30 min for the whole sensor to revert to its original state (Figure S18). A similar phenomenon was witnessed for the fumed sensor obtained from the PA leakage model as well (Figure S19). Furthermore, as depicted in Figure 4c, reversible changes in both color and emission could be observed for the printing sensor under daylight and UV light via repeated cycling fuming or staining. Such a good reusability made CMVMN more practical in real applications.

In-situ probe of biomolecules was the final and crucial demand for bioconjugations. So, after verifying the high selectivity of CMVMN towards primary amines, we moved forward to study its behavior in aqueous solution with biomolecules. At first, numerous small biomolecules were chosen and cultured with CMVMN. As shown in Figure 5a, nearly no fluorescence enhancement was detected of CMVMN in the presence of other polar basic (histidine), polar neutral (tryptophan, serine, asparagine and cysteine), polar acidic (glutamic acid) and 

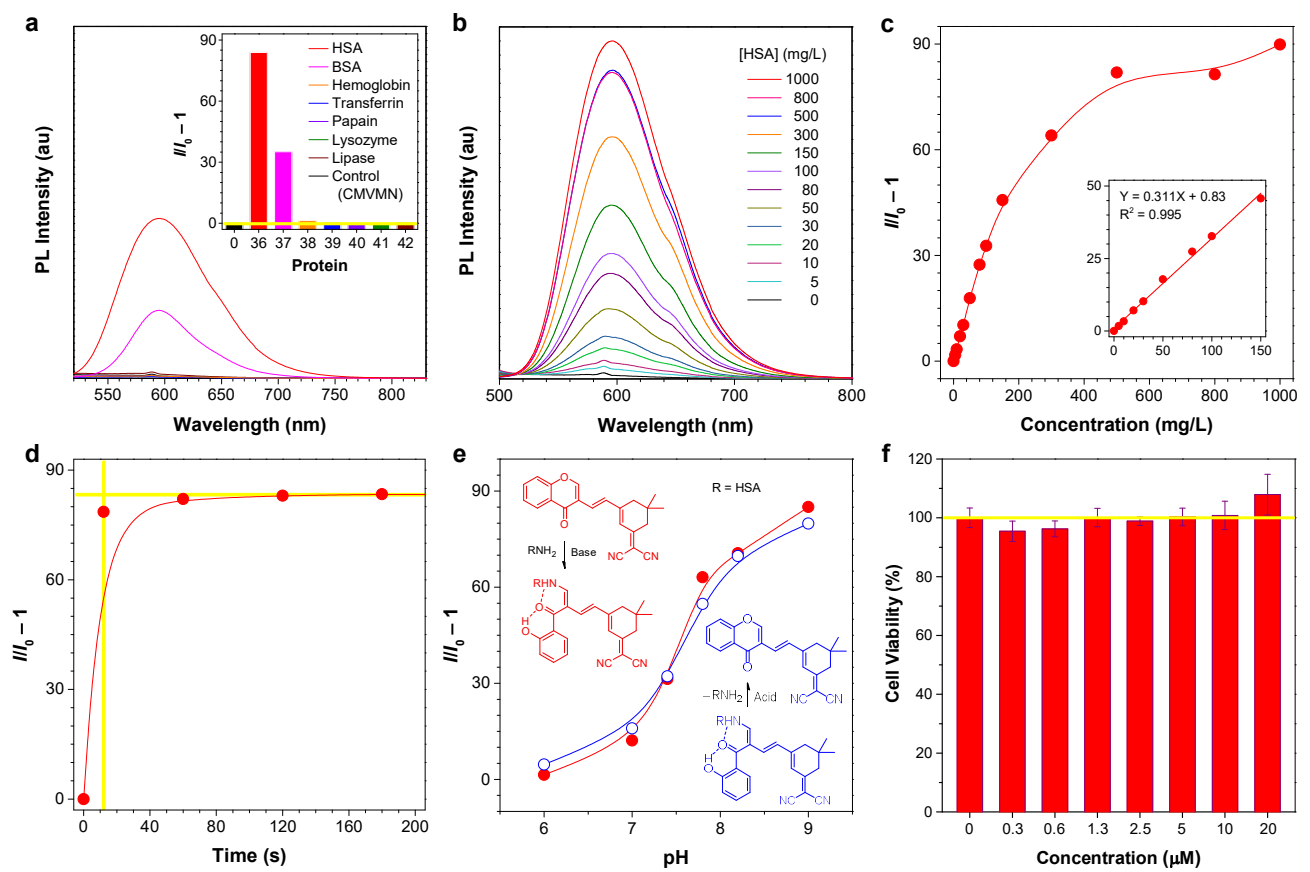

Figure 6. (a) PL spectra and (inset) relative fluorescence intensity $\left(I / I_{0}-1\right)$ of CMVMN $(10 \mu \mathrm{M})$ in the presence of various protein $(1 \mathrm{mg} / \mathrm{mL})$ in PBS. Protein: $[(0) \mathrm{CMVNM}$ (control)], (36) HSA, (37) BSA, (38) hemoglobin, (39) transferrin, (40) papain, (41) lysozyme, (42) lipase. (b) Change in PL spectrum of CMVMN upon addition of different concentration of HSA. (c) Plot of $\left(I / I_{0}-1\right)$ vs. concentration of HSA [HSA]. Inset: Linear fitting curve of $\left(I / I_{0}-1\right)$ toward [HSA]. (d) Time course of amination of CMVMN with HSA. (e) Effect of $\mathrm{pH}$ on the reaction of CMVMN with HSA: amination at high pH and de-amination at low pH. (f) Effect of CMVMN on the cell viability of A375. Data shown were the mean \pm standard deviation of five separate measurements. $\lambda_{\mathrm{ex} / \mathrm{em}}=480 / 595 \mathrm{~nm}$.

nonpolar hydrophobic (alanine) amino acids as well as glutathione (an important tripeptide serves as an antioxidant and free radical scavenger). ${ }^{31}$ In terms of the structural formula, only arginine and lysine possessed reactive residues with the $\mathrm{NH}_{2}$ group, and the guanidino moiety was called the primary guanidino amine. ${ }^{32}$ Also, it's worth noting that the $\alpha$-amino groups of amino acids were usually less reactive in nucleophilic bioconjugation due to the interference of the carboxylic acid. ${ }^{12 c, 33}$ Thus, the enhanced fluorescence detected for lysine and arginine should be attributed to the high reactivity of CMVMN with primary amine groups. Besides, $N(\alpha)$-Boc- $L$-arginine was tested to verify the reaction site of arginine and we witnessed a similar fluorescence response towards CMVMN as arginine, proving the importance of the primary guanidino amine for the amination (Figure S20). In additional, nitrogen bases (thymine, adenine and cytosine) and monosaccharides (glucose and fructose) were tested, but CMVMN was found to be inert to these biomolecules. Thus, even though CMVMN displayed a very weak reactivity towards primary amines in aqueous solution it retained the ability to selectively aminate with primary amine groups. The selectivity of CMVMN towards specific amino acids (lysine and arginine) could further make it a promising candidate for selective labeling of proteins.

Next, the amination process in different $\mathrm{pH}$ was investigated. To avoid the deterioration of the activity and structure of biomolecules, mild $\mathrm{pH}$ conditions were preferred. Thus, the fluorescence response of CMVMN to arginine or lysine in $\mathrm{aH}$ range from 6.0 to 9.0 was studied. As depicted in Figure5b, CMVMN reacted readily with arginine and lysine under alkaline conditions. However, the amination was greatly inhibited at $\mathrm{pH}$ 6.0. Likely, a tunable amination process between CMVMN and primary amines could be achieved under mild $\mathrm{pH}$ conditions. Meanwhile, a dramatic change in relative fluorescence intensity was observed from $\mathrm{pH} 7.0$ to 7.8 , which suggested that CMVMN might be lighted up in a relative alkaline environment like in the mitochondria of cells. In the presence of CMVMN alone, the relative fluorescence intensity kept almost constant at different $\mathrm{pH}$ and such a stability was favorable for sensing and labeling (Figure S21). Another worth noting thing is the ability of CMVMN for differentiation primary amines from secondary amines. When $\mathrm{N}_{6}$-methyl-L-lysine (Me-lys), the main product for lysine after methylation, was picked up as a model compound to replace some lysine in the same amount (the amount added was defined as methylation ratio, $f_{\text {Me-lys }}$ ), an decrease in the relative fluorescence intensity was detected. A good linearity between the relative fluorescence intensity and $f_{\text {Me-lys }}$ was found as well, which demonstrated the applicability of CMVMN for selective amination of primary amines for biomolecules.

Followed, the application of protein labeling was performed. Upon addition of HSA, the fluorescence spectrum largely intensified and the relative fluorescence intensity was increased by 90 folds (Figure 6a). Such an enhancement effect for HSA was noticed to be surprisingly stronger than that of arginine or lysine, especially when the high concentration $(10 \mathrm{mM})$ used for amino acids testing was taken into account. Prior to this, AlEgens were found to be more reactive in the cavity due to the restriction of their intramolecular motions. ${ }^{34}$ In view of the hydrophobic nature of CMVMN, it may be trapped in the cavity of HSA to result in such a significantly enhanced fluorescent signal. At least, the enhanced specificity could make CMVMN more applicable for in-situ labeling of biomacromolecules since interference from other small biomolecules became negligible. Identically, because of the difference in the cavity, it was reasonable to see a discrepant selectivity of CMVMN towards other proteins, including BSA, hemoglobin, transferrin, papain, lysozyme and lipase (Figure 6a).

To get further insight into such protein selectivity, molecular docking calculations among the proteins and CMVMN were 
performed. In the HSA-CMVMN complex, CMVMN was docked in the hydrophobic cleft via the hydrogen bond interaction with ILE-142 (Figure S22). Further, nearest neighbor residues around the ligand were identified and the ARG-117 could be the reaction site in the binding cleft. In the BSA-CMVMN complex, two binding sites were found and both sites exhibited arginine residues around the ligand. However, one of the binding sites was not activated due to the hydrogen bond interactions which locked the aromatic ring and thereby hindered the formation of the transition state (Figure S23). That explained why CMVMN could label BSA but its reactivity was not as good as HSA. For the same reason, hemoglobin was found to be not reactive with CMVMN (Figure S24). Meanwhile, other proteins (transferrin, papain lysozyme, and lipase) were found not only unable to form stable complex with CMVMN, but also none of the arginine or lysine exhibit around the possible binding sites (Figure S25-S28) On the other hand, the reaction solution of CMVMN and HSA was collected and it showed an absorption peak at around 487 $\mathrm{nm}$ (Figure S29), which was in accordance with that of the amination products of CMVMN (Figure 2a). Thus, it verified the efficient recognition and capture of primary amino groups inside HSA by CMVMN. But even though the amination of CMVMN with specific amino acids (lysine and arginine) could be applied for protein labeling, its efficiency is highly associated with the hydrophobic cleft of proteins. This may offer a new approach for specific protein labeling.

Subsequently, a quantitative analysis of HSA was performed. As depicted in Figure $6 \mathrm{~b}$, the fluorescence of CMVMN was gradually increased upon addition of HSA and a good linear curve was obtained at a concentration range of $0-150 \mathrm{mg} / \mathrm{L}$ (Figure 6c), from which a detection limit of $1.2 \mathrm{mg} / \mathrm{L}$ was calculated. Quantitative analysis of BSA was realized (Figure S30) as well. Furthermore, we studied the reaction kinetics of CMVMN with HSA. As shown in Figure 6d, a fluorescence "turnon" response occurred within $10 \mathrm{~s}$ and the relative fluorescence intensity rose quickly and nearly reached a plateau. The fast rate of CMVMN in biolabeling was indeed advantageous for bioconjugation in-situ. Also, a $\mathrm{pH}$-dependent reactivity to HSA was detected (Figure 6e), which was similar to that of lysine and arginine. More excitingly, when lowering the $\mathrm{pH}$ of the reaction solution, the relative fluorescence intensity dropped rapidly and its value was close to that of the original state (Figure 6e), suggesting of the occurrence of the deamination. In other words, tunable biolabeling of proteins in physiological $\mathrm{pH}$ conditions was realized by CMVMN and the molecule could be further applied for damage-free dynamic monitoring of proteins in-situ. The cytotoxicity of CMVMN to A375 cells and $\mathrm{C} 2 \mathrm{C} 12$ cells was tested by MTT assay and no considerable influence on the viability of cells was detected after $24 \mathrm{~h}$ incubation (Figure $6 \mathrm{f}$ and Figure S31).

On account of the low cytotoxicity of CMVMN, the cell imaging experiment for CMVMN was proceeded. A bright fluorescence of CMVMN was observed in human melanoma cell line A375 cells within $5 \mathrm{~min}$ and the fluorescence intensity reached a plateau after $15 \mathrm{~min}$ of incubation (Figure 7a). This indicated the good biocompatibility and high reactivity of CMVMN in cells. The colocalization experiments with Mito-tracker green were also conducted and the image of cells stained with CMVMN merged well with the one labeled by Mito-tracker green. The Pearson's
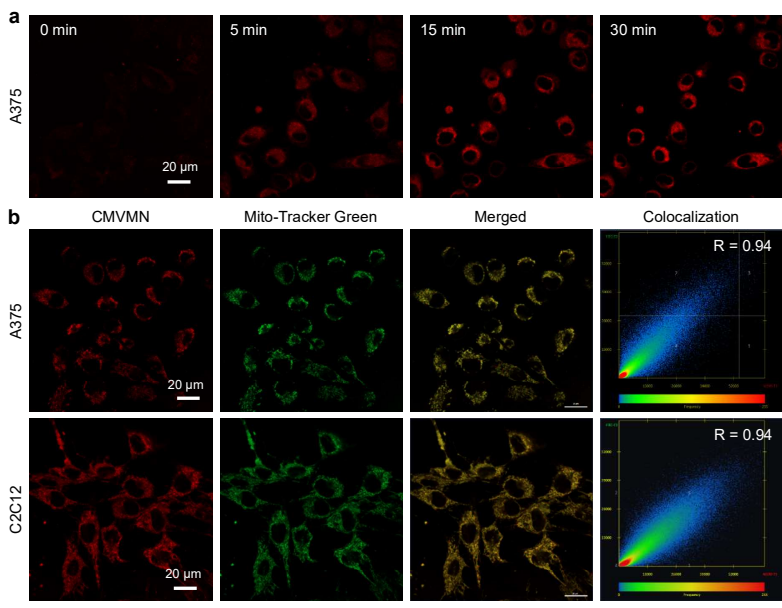

Merged

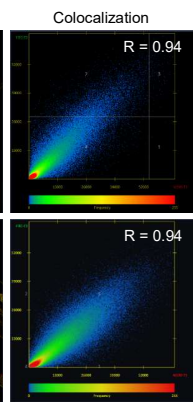

Figure 7. (a) Fluorescence images of $A 375$ cells incubated with CMVMN (10 $\mu \mathrm{M})$. (b) Confocal microscopic images of A375 cells and C2C12 cells stained with CMVMN $(10 \mu \mathrm{M}))$ and colocalization with Mito - Tracker Green $(1 \mu \mathrm{M})$. R $=$ Pearson's coefficient.

co-localization coefficient was calculated to be 0.94 (Figure $7 \mathrm{~b}$ ), which suggested that CMVMN is predominantly lighted up in mitochondria. A similar phenomenon was observed for mouse skeletal muscle cell line $\mathrm{C} 2 \mathrm{C} 12$ cells as well (Figure $7 \mathrm{~b}$ and Figure S32), indicating the potential of CMVMN for mitochondria-staining. Since the amination process of CMVMN was $\mathrm{pH}$-dependent and mitochondria happened to be slightly alkaline ${ }^{35}$ CMVMN was partially astricted in mitochondria via amination and then lighted up.

In view of the high reactivity between CMVMN and HSA, it becomes something notable for the performance of CMVMN in cells with abundant HSA. A variety of major plasma proteins, including albumin, were reported to be secreted in liver cells. ${ }^{36}$ Then, the existence of HSA in cytoplasmic matrix and other organelles would induce a relatively high fluorescent signal outside of mitochondria for liver cells. Curiously, we conducted co-localization experiments with Mito-tracker green for human hepatocarcinoma cell line HepG2 cells and normal liver cell line L-02 cells. To have a better vision of the co-localization efficiency, 3D z-stack imaging was obtained. As shown in Figure S33, the calculated Pearson's co-localization coefficient was good for $\mathrm{C} 2 \mathrm{C} 12$ cells $(0.90)$ once again. However, it became attenuated but tolerable (0.69-0.71) for liver cells, indicating the amination of CMVMN with HSA in liver cells. On the other hand, human lung adenocarcinoma cell line A549 cells was also tested and resulted in a good mitochondria-staining ability as expected. Hence, the $\mathrm{pH}$-dependent reactivity of CMVMN made it a promising mitochondria-staining probe and other reactive proteins like HSA did exist in mitochondria for CMVMN. On the other hand, the fluorescence intensity in liver cells (HepG2 cells and L-02 cells) was found to be not significantly enhanced compared with that of $\mathrm{C} 2 \mathrm{C} 12$ cells and A549 cells (Figure S34), and the mitochondria isolated from $\mathrm{C} 2 \mathrm{C} 12$ cells induced an obvious enhancement in fluorescent signal after mixing with CMVMN (Figure S35), which further verified our conjecture of the existence of reactive proteins in mitochondria for CMVMN.

However, in previous works, special functional groups like triphenylphosphonium salt and pyridinium were needed to incorporate into luminogens to make them positively charged to target the mitochondria. ${ }^{37}$ Thus, CMVMN was a neutral molecule 
with mitochondria staining ability. Undoubtedly, it provided a new paradigm for designing mitochondria staining probes.

\section{Conclusion}

The activated chromone derivative CMVMN reported in this paper filled the gap of selective bioconjugation for native primary amine groups. Its reaction with primary amines was fast, efficient and highly selective in both solution and cells, and could be followed by the intense fluorescence "turn-on" signal. The combination of experimental results and theoretical calculations showed that both steric effect and the intramolecular hydrogen bonding between the carbonyl and $\mathrm{NH}$ groups were the cause for the high selectivity of CMVMN towards primary amines. Also, the AIE-active property of CMVMN made it suitable for solidstate sensing of amine vapors and printing process with a good performance in PA diffusion and leakage model. Moreover, CMVMN displayed a good mitochondria-staining property due to its $\mathrm{pH}$-dependent reactivity with primary amines, which provided a new insight into the design of organelle-staining probes. To our knowledge, CMVMN was an example of scarcity for selective bioconjugation of primary amines in-situ and it was also a neutral molecule with mitochondria-staining ability. It was anticipated that the present results would not only promote the development of chemoselective reactions in chemistry, but also offers new possibilities in biology.

\section{Experimental Section}

Experimental Details please see supporting information.

Materials and methods, synthetic procedures, crystallographic data, characterizations, photophysical data and imaging data (PDF)

X-ray crystallographic information (CIF)

Video S1: The luminescence of amorphous powder and crystalline solid of CMVMN became stronger after cooling with liquid nitrogen (MP4)

Video S2: The color of "spider-web" sensor changed upon exposure to PA vapor (MP4)

Video S3: The luminescence of "spider-web" sensor changed upon exposure to PA vapor (MP4)

Video S4: The color of "spider-web" sensor changed in PA leakage model (MP4)

Video S5: The luminescence of "spider-web" sensor changed in PA leakage model (MP4)

\section{Acknowledgements}

We are grateful for financial support from the National Natural Science Foundation of China (21788102), the Research Grants Council of Hong Kong (16307020, 16306620, 16305518, NHKUST609/19, C6009-17G, and C6014-20w), the Innovation and Technology Commission (ITC-CNERC14SC01 and ITCPD/17-9), and the Natural Science Foundation of Guangdong Province (201913121205002).

Keywords: bioconjugation - primary amines $\cdot$ aggregationinduced emission $\bullet$ protein labelling $\bullet$ mitochondria-staining
[1] a) W. P. Kloosterman, E. Wienholds, E. de Bruijn, S. Kauppinen, R. Plasterk, Nat. Methods 2006, 3, 27-29; b) H. W. Liu, K. Li, X. X. Hu, L. Zhu, Q. Rong, Y. Liu, X. B. Zhang, J. Hasserodt, F. L. Qu, W. Tan, Angew. Chem. Int. Ed. 2017, 56, 11788-11792; c) S. S. Nguyen, J. A Prescher, Nat. Rev. Chem. 2020, 4, 476-489; d) A. Borrmann, J. C. M. van Hest, Chem. Sci. 2014, 5, 2123-2134.

[2] a) J. Lippincott-Schwartz, G. H. Patterson, Science 2003, 300, 87-91 b) J. Zhang, R. Campbell, A. Ting, R. Tsien, Nat. Rev. Mol. Cell Biol. 2002, 3, 906-918; c) G. U. Nienhaus, Angew. Chem. Int. Ed. 2008, 47, 8992-8994.

[3] J. A. Prescher, C. R. Bertozzi, Nat. Chem. Biol. 2005, 1, 13-21.

[4] H. C. Hang, C. Yu, D. L. Kato, C. R. Bertozzi, Proc. Natl. Acad. Sci. U.S.A. 2003, 100, 14846-14851.

[5] a) E. M. Sletten, C. R. Bertozzi, Angew. Chem. Int. Ed. 2009, 48, 69746998; b) R. K. V. Lim, Q. Lin, Sci. China Chem. 2010, 53, 61-70; c) X. Fan, J. Li, P. R. Chen, Natl. Sci. Rev. 2017, 4, 300-302.

[6] a) D. M. Patterson, L. A. Nazarova, B. Xie, D. N. Kamber, J. A Prescher, J. Am. Chem. Soc. 2012, 134, 18638-18643; b) L. JosaCulleré, Y. A. Wainman, K. M. Brindle, F. J. Leeper, RSC Adv. 2014, 4, 52241-52244; c) J. Luo, Q. Liu, K. Morihiro, A. Deiters, Nat. Chem. 2016, 8, 1027-1034; d) J. Li, P. R. Chen, Nat. Chem. Biol. 2016, 12, 129-137; e) Y. Tian, Q. Lin, ACS Chem. Biol. 2019, 14, 2489-2496; f) Y. Li, Z. Lou, H. Li, H. Yang, Y. Zhao, H. Fu, Angew. Chem. Int. Ed. 2020, 59, 3671-3677; g) R. Dzijak, J. Galeta, A. Vázquez, J. Kozák, M Matoušová, H. Fulka, M. Dračínský, M. Vrabel, JACS Au 2021, 1(1) 23-30; h) M. L. W. J. Smeenk, J. Agramunt, K. M. Bonger, Curr. Opin Chem. Biol. 2021, 60, 79-88; i) K. Porte, M. Riberaud, R. Châtre, D Audisio, S. Papot, F. Taran, ChemBioChem 2021, 22, 100-113.

[7] a) M. Grammel, H. C. Hang, Nat. Chem. Biol. 2013, 9, 475-484; b) C. P Ramil, Q. Lin, Chem. Commun. 2013, 49, 11007-11022.

[8] a) T. Peng, H. C. Hang, J. Am. Chem. Soc. 2016, 138(43), 1442314433; b) A. Godinat, A. A. Bazhin, E. A. Goun, Drug Discov. Today 2018, 23, 1584-1590.

[9] J. L. Klockow, K. S. Hettie, T. E. Glass, Comprehensive Supramolecular Chemistry II Elsevier 2017, 447-467.

[10] a) J. H. Flanagan, S. H. Khan, S. Menchen, S. A. Soper, R. P. Hammer Bioconjugate Chem. 1997, 8, 751-756; b) G. Barbarella, Chem. Eur. J. 2002, 8, 5072-5077; c) A. R. Morales, K. J. Schafer-Hales, A. I. Marcus, K. D. Belfield, Bioconjugate Chem. 2008, 19, 2559-2567; d) J. Xu, C. Zeng, W. Chu, F. Pan, J. M. Rothfuss, F. Zhang, Z. Tu, D. Zhou, D Zeng, S. Vangveravong, F. Johnston, D. Spitzer, K. C. Chang, R. S Hotchkiss, W. G. Hawkins, K. T. Wheeler, R. H. Mach, Nat. Commun. 2011, 2, 380; e) R. Jia, W. Tian, H. Bai, J. Zhang, S. Wang, J. Zhang, Nat. Commun. 2019, 10, 795; f) V. G. Amorim, S. M. G. Melo, R. F. Leite, P. A. Coutinho, S. M. P. da Silva, A. R. Silva, F. G. Amorim, R. G W. Pires, J. B. Coitinho, F. S. Emery, L. C. D. Rezende, Dyes Pigm. 2020, 182, 108646.

[11] a) B. J. C. Quah, H. S. Warren, C. R. Parish, Nat. Protoc. 2007, 2 , 2049-2056; b) T. Tanaka, K. Tainaka, T. Kamei, A. Okamoto, J. Am. Chem. Soc. 2007, 129, 5612-5620; c) D. Wang, J. Fan, X. Gao, B Wang, S. Sun, X. Peng, J. Org. Chem. 2009, 74, 7675-7683; d) L. Zhang, Y. Cheng, J. Lei, Y. Liu, Q. Hao, H. Ju, Anal. Chem. 2013, 85, 8001-8007; e) L. J. Xia, X. F. Guo, Y. Ji, L. Chen, H. Wang, Anal. Methods 2018, 10, 3188-3196.

[12] a) M. Adamczyk, J. R. Fishpaugh, K. J. Heuser, Bioconjugate Chem. 1997, 8, 253-255; b) H. Zhao, P. Theato, Polym. Chem. 2013, 4, 891894; c) S. Jeon, T. I. Kim, H. Jin, U. Lee, J. Bae, J. Bouffard, Y. Kim, J. Am. Chem. Soc. 2020, 142, 9231-9239.

[13] a) B. K. Wetzl, S. M. Yarmoluk, D. B. Craig, O. S. Wolfbeis, Angew. Chem. Int. Ed. 2004, 43, 5400-5402; b) G. J. Mohr, Anal. Bioanal. Chem. 2006, 386, 1201-1214; c) A. R. Longstreet, M. Jo, R. R. Chandler, K. Hanson, N. Zhan, J. J. Hrudka, H. Mattoussi, M. Shatruk, D. T. McQuade, J. Am. Chem. Soc. 2014, 136, 15493-15496; d) A. M. EISohly, M. B. Francis, Acc. Chem. Res. 2015, 48, 1971-1978; e) A. D. Guo, D. Wei, H. J. Nie, H. Hu, C. Peng, S. T. Li, K. N. Yan, B. S. Zhou, L. Feng, C. Fang, M. Tian, R. Huang, X. H. Chen, Nat. Commun. 2020, 11,5472 .

[14] a) B. He, H. Su, T. Bai, Y. Wu, S. Li, M. Gao, R. Hu, Z. Zhao, A. Qin, J. Ling, B. Z. Tang, J. Am. Chem. Soc. 2017, 139, 5437-5443; b) X. Hu, X. 
Zhao, B. He, Z. Zhao, Z. Zheng, P. Zhang, X. Shi, R. T. K. Kwok, J. W. Y. Lam, A. Qin, B. Z. Tang, Research 2018, 3152870.

[15] a) V. A. Zagorevskii, É. K. Orlova, I. D. Tsvetkova, V. G. Vinokurov, V. S. Troitskaya, S. G. Rozenberg, Chem. Heterocycl. Compd. 1971, 7 675-680; b) G. P. Ellis, I. M. Lockhart, Wiley-VCH: New York, 2007, 31 $1-1196$

[16] D. S. Kemp, G. Hanson, J. Org. Chem. 1981, 46, 4971-4975

[17] a) P. J. L. Bell, P. Karuso, J. Am. Chem. Soc. 2003, 125, 9304-9305; b) D. R. Coghlan, J. A. Mackintosh, P. Karuso, Org. Lett. 2005, 7 , 2401-2404.

[18] a) V. Y. Sosnovskikh, V. S. Moshkin, M. I. Kodess, Tetrahedron Lett. 2009, 50, 6515-6518; b) V. O. laroshenko, S. Mkrtchyan, A. Gevorgyan T. Grigoryan, A. Villinger, P. Langer, RSC Adv. 2015, 5, 28717-28724; c) A. S. Badran, N. M. El-Gohary, M. A. Ibrahim, S. H. Hashiem, J. Heterocycl. Chem. 2020, 57, 2570-2585.

[19] a) D. Wu, A. C. Sedgwick, T. Gunnlaugsson, E. U. Akkaya, J. Yoon, T. D. James, Chem. Soc. Rev. 2017, 46, 7097-7472; b) D. Hu, T. Zhang S. Li, T. Yu, X. Zhang, R. Hu, J. Feng, S. Wang, T. Liang, J. Chen, L. N Sobenina, B. A. Trofimov, Y. Li, J. Ma, G. Yang, Nat. Commun. 2018, 9 362; c) D. Cao, Z. Liu, P. Verwilst, S. Koo, P. Jangjili, J. S. Kim, W. Lin, Chem. Rev. 2019, 119, 10403-10519; d) Y. Zhang, X. Wang, X. Bai, P. Li, D. Su, W. Zhang, W. Zhang, B. Tang, Anal. Chem. 2019, 91, 85918594.

[20] D. Ding, K. Li, B. Liu, B. Z. Tang, Acc. Chem. Res. 2013, 46, $2441-$ 2453.

[21] J. Luo, Z. Xie, J. W. Lam, L. Cheng, H. Chen, C. Qiu, H. S. Kwok, X. Zhan, Y. Liu, D. Zhu, B. Z. Tang, Chem. Commun. 2001, 18, 17401741.

[22] a) J. Liang, B. Z. Tang, B. Liu, Chem. Soc. Rev. 2015, 44, 2798-2811; b) J. Mei, Y. Huang, H. Tian, ACS Appl. Mater. Interfaces 2018, 10 12217-12261; c) S. Gao, G. Wei, S. Zhang, B. Zheng, J. Xu, G. Chen, M. Li, S. Song, W. Fu, Z. Xiao, W. Lu, Nat. Commun. 2019, 10, 2206 d) Q. Guan, N. Li, L. Shi, C. Yu, X. Gao, J. Yang, Y. Guo, P. Li, X. Zhu, ACS Biomater. Sci. Eng. 2019, 5, 3618-3630; e) J. Yang, M. Fang, Z. Li, Aggregate 2020, 1, 6-18; f) Z. Liu, Q. Wang, Z. Zhu, M. Liu, X. Zhao W. H. Zhu, Chem. Sci. 2020, 11, 12755-12763; g) H. Li, H. Kim, J. Han, V. Nguyen, X. Peng, J. Yoon, Aggregate 2021, 2, e51.

[23] Y. Dong, J. W. Lam, A. Qin, Z. Li, J. Sun, H. H. Sung, I. D. Williams, B. Z. Tang, Chem. Commun. 2007, 40-42.

[24] a) M. Yamaguchi, S. Ito, A. Hirose, K. Tanaka, Y. Chujo, Mater. Chem Front. 2017, 1, 1573-1579; b) Z. Wang, T. Wang, C. Zhang, M. G. Humphrey, Phys. Chem. Chem. Phys. 2017, 19, 12928-12935; c) N. Nirmalananthan, T. Behnke, K. Hoffmann, D. Kage, C. F. Gers-Panther, W. Frank, T. J. J. Müller, U. Resch-Genger, J. Phys. Chem. C 2018 122, 11119-11127; d) S. Liu, Y. Li, H. Zhang, Z. Zhao, X. Lu, J. W. Y. Lam, B. Z. Tang, ACS Materials Lett. 2019, 1, 425-431; e) Z. Yu, C. Yu Q. Wu, H. Wang, L. Jiao, W. Y. Wong, E. Hao, J. Mater. Chem. C 2019 7, 4533-4542.

[25] Z. Zhao, H. Zhang, J. W. Y. Lam, B. Z. Tang, Angew. Chem. Int. Ed. 2020, 59, 9888-9907.

[26] W. Chen, A. Pacheco, Y. Takano, J. J. Day, K. Hanaoka, M. Xian, Angew. Chem., Int. Ed. 2016, 55, 9993-9996.

[27] a) H. Si, K. Wang, B. Song, B. Z. Tang, Polym. Chem. 2020, 11, 25682575; b) A. O. Konuray, X. Fernández-Francos, X. Ramis, Polym. Chem. 2020, 8, 5934-5947.

[28] a) S. Singha, Y. W. Jun, S. Sarkar, K. H. Ahn, Acc. Chem. Res. 2019 , 52, 2571-2581; b) H. Liu, S. Wang, H. Gao, Z. Shen, Eur. J. Org. Chem. 2020, 52, 5647-5663.

[29] a) L. Shi, Y. Fu, C. He, D. Zhu, Y. Gao, Y. Wang, Q. He, H. Cao, J. Cheng, Chem. Commun. 2014, 50, 872-874; b) Y. Fu, W. Xu, Q. He, J. Cheng, Sci. China Chem. 2016, 59, 3-15; c) Y. Jiang, Z. Zhong, W. Ou H. Shi, P. Alam, B. Z. Tang, J. Qin, Y. Tang, Food Chem. 2020, 327 127056.

[30] A. Gupta, ChemistrySelect 2019, 4, 12848-12860.

[31] a) C. C. Winterbourn, Arch. Biochem. Biophys. 2016, 595, 68-71; b) X. L. Liu, L. Y. Niu, Y. Z. Chen, M. L. Zheng, Y. Yang, Q. Z. Yang, Org Biomol. Chem. 2017, 15, 1072-1075.

[32] E. C. Vermisoglou, P. Jakubec, A. Bakandritsos, V. Kupka, M. Pykal, V. Šedajová, J. Vlček, O. Tomanec, M. Scheibe, R. Zbořil, M. Otyepka, ChemSusChem 2021, 14, 3904-3914.
[33] a) O. Koniev, A. Wagner, Chem. Soc. Rev. 2015, 44, 5495-5551; b) J N. deGruyter, L. R. Malins, P. S. Baran, Biochemistry 2017, 56, 38633873; c) V. Grundler, K. Gademann, ACS Med. Chem. Lett. 2014, 5, 1290-1295

[34] P. Wei, Z. Li, J. X. Zhang, Z. Zhao, H. Xing, Y. Tu, J. Gong, T. S. Cheung, S. Hu, H. H. Y. Sung, I. D. Williams, R. T. K. Kwok, J. W. Y Lam, B. Z. Tang, Chem. Mater. 2019, 31, 1092-1100.

[35] a) J. Casey, S. Grinstein, J. Orlowski, Nat. Rev. Mol. Cell Biol. 2010, 11 50-61; b) M. Benčina, Sensors 2013, 13, 16736-16758.

[36] a) R. Zhao, T. Jia, H. Shi, C. Huang, J. Mater. Chem. B 2019, 7, 2782 2789; b) T. Peters, C. B. Anfinsen, J. Biol. Chem. 1950, 186, 805-813; c) G. J. van der Vusse, Drug Metab. Pharmacokinet. 2009, 24, 300307.

[37] a) Q. Hu, M. Gao, G. Feng, B. Liu, Angew. Chem. Int. Ed. 2014, 53 14225-14229; b) A. R. Sarkar, C. H. Heo, L. Xu, H. W. Lee, H. Y. Si, J W. Byun, H. M. Kim, Chem. Sci. 2016, 7, 766-773; c) Z. Lv, Z. Man, H. Cui, Z. Xu, H. Cao, S. Li, Q. Liao, Q. He, L. Zheng, H. Fu, Adv. Funct Mater. 2021, 31, 2009329; d) N. E. Choi, J. Y. Lee, E. C. Park, J. H. Lee, J. Lee, Molecules 2021, 26, 217. 